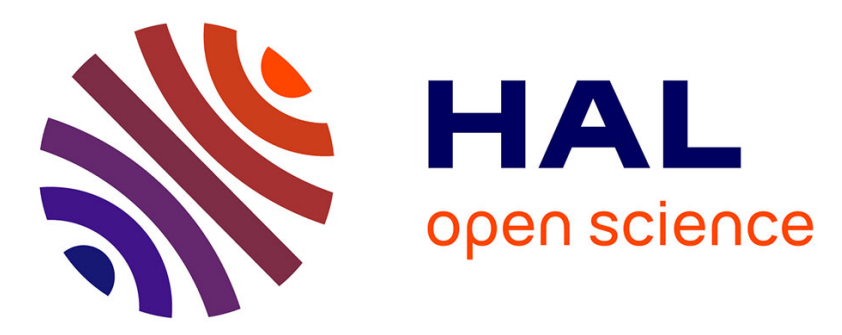

\title{
Estimate of the travelling wave speed for an integro-differential equation
}

Nikolay Bessonov, Anne Beuter, Sergei Trofimchuk, Vitaly Volpert

\section{To cite this version:}

Nikolay Bessonov, Anne Beuter, Sergei Trofimchuk, Vitaly Volpert. Estimate of the travelling wave speed for an integro-differential equation. Applied Mathematics Letters, 2019, 88, pp.103-110. 10.1016/j.aml.2018.07.037 . hal-01941455

\section{HAL Id: hal-01941455 \\ https://hal.science/hal-01941455}

Submitted on 19 Oct 2021

HAL is a multi-disciplinary open access archive for the deposit and dissemination of scientific research documents, whether they are published or not. The documents may come from teaching and research institutions in France or abroad, or from public or private research centers.
L'archive ouverte pluridisciplinaire HAL, est destinée au dépôt et à la diffusion de documents scientifiques de niveau recherche, publiés ou non, émanant des établissements d'enseignement et de recherche français ou étrangers, des laboratoires publics ou privés. 


\title{
Estimate of the travelling wave speed for an integro-differential equation
}

\author{
N. Bessonov ${ }^{1}$, A. Beuter ${ }^{2}$, S. Trofimchuk ${ }^{3}$, V. Volpert ${ }^{4,5,6,7}$ \\ ${ }^{1}$ Institute of Problems of Mechanical Engineering, Russian Academy of Sciences \\ 199178 Saint Petersburg, Russia \\ ${ }^{2}$ Bordeaux INP, Bordeaux, France, Equipage Innovation SARL, Plérin, France \\ ${ }^{3}$ Instituto de Matematica y Fisica, Universidad de Talca, Casilla 747, Talca, Chile \\ ${ }^{4}$ Institut Camille Jordan, UMR 5208 CNRS, University Lyon 1, 69622 Villeurbanne, France \\ ${ }^{5}$ INRIA Team Dracula, INRIA Lyon La Doua, 69603 Villeurbanne, France \\ ${ }^{6}$ Peoples Friendship University of Russia (RUDN University) \\ 6 Miklukho-Maklaya St, Moscow, 117198, Russian Federation \\ 7 Poncelet Center, UMI 2615 CNRS, 11 Bolshoy Vlasyevskiy, 119002 Moscow \\ Russian Federation
}

\begin{abstract}
Travelling waves for nonlocal reaction-diffusion equations are studied. The minimax representation of the wave speed is obtained. It is used to obtain analytical estimates and asymptotic values of the speed. Two regimes of wave propagation are identified. One of the them is dominated by diffusion and another one by the nonlocal interaction.
\end{abstract}

Key words: nonlocal reaction-diffusion equation, wave speed, minimax representation, estimates

\section{Introduction}

We consider the nonlocal reaction-diffusion equation

$$
\frac{\partial u}{\partial t}=D \frac{\partial^{2} u}{\partial x^{2}}+\int_{-\infty}^{\infty} \phi(x-y) S(u(y+a, t-\tau)) d y-k u,
$$

where $\phi(x)$ is a non-negative integrable function with a bounded support, $S(u)$ is a smooth non-negative and non-decreasing function such that the equation

$$
S(u)=k u
$$


has three solutions, $u_{+}<u_{0}<u_{-}$, and $S^{\prime}\left(u_{ \pm}\right)<k$. Some additional conditions and examples will be considered below. The diffusion coefficient $D$ can be positive or zero, $\tau \geq 0, a$ is a real number. This equation describes various applications including propagation of neural waves $[1,2]$. In this case $S(u)$ is neuron response function, $\phi(x)$ the connectivity function showing how the connection between neurons depends on the distance between them, time delay $\tau$ is an average time of signal transmission between connected neurons. A positive constant $k$ characterizes the rate of signal decay, and a real number $a$ is introduced for convenience, it will be used in the analysis of the problem.

Equation (1.1) is considered on the whole axis. We will be interested in traveling waves solutions of this equations introduced in Section 2 below. One of the important their characteristics is the speed of wave propagation. Though, in general, it cannot be found explicitly, various various minimax and variational representations of the wave speed can be used to get some estimates and to study its asymptotics. The first mini-max representation of the wave speed was suggested in [3] for the scalar equation on the basis of the phase plane analysis. A similar approach was used in [4] for a system of two equations in combustion theory. Another mini-max representation, based on the global stability of travelling waves, was developed for monotone reaction-diffusion systems (see [5] and the references therein). It was used to estimate the speed of blood coagulation waves in [6]. This approach was generalized for some integro-differential equations $[7,8]$. Let us also mention variational representations of the wave speed obtained in $[9,10]$. In this work, we will use the method based on the global stability argument in order to obtain a minimax representation of the wave speed for another integro-differential equation. We will use it to get analytical estimates and asymptotic values of the speed.

\section{Minimax representation of the wave speed in the monotone case}

Profile $w(x)$ of a travelling wave solution $u(x, t)=w(x-c t)$ of equation (1.1) is a solution of the problem

$$
\begin{gathered}
D w^{\prime \prime}+c w^{\prime}+\int_{-\infty}^{\infty} \phi(x-y) S(w(y+a+c \tau)) d y-k w=0 \\
w( \pm \infty)=u_{ \pm}
\end{gathered}
$$

Existence and global stability of travelling wave solutions of integro-differential equations are studied under various conditions on the functions $\phi(x), S(w)$, and constants $D>0$ or $D=0, \tau>0$ or $\tau=0[1,7,11,12]$. Moreover, these waves are monotonically decreasing functions of $x$.

The wave is globally asymptotically stable if the following convergence

$$
\sup _{x \in \mathbb{R}}|u(x, t)-w(x-c t-h)| \rightarrow 0, \quad t \rightarrow \infty
$$


occurs for some constant $h$ and for the solution $u(x, t)$ of equation (1.1) considered on the whole axis with the initial condition $u(x, t)=u_{0}(x),-\tau \leq t \leq 0$. Here $u_{0}(x)$ is a continuous decreasing function with the limits $u_{0}( \pm \infty)=u_{ \pm}$. Note that more general stability results are available. This assumption is sufficient for what follows. If the function $S(u)$ is increasing and $\phi(x)$ is non-negative, then the wave stability follows from the results of the work [12] (see also [11] for the nonlocal equation without delay). We suppose these conditions to be satisfied.

Proposition 2.1. Suppose that $\tau=0, S(u)$ is increasing, and there exists a monotonically decreasing solution $w(x)$ of problem $(2.1),(2.2)$. Then for any function $\rho(x)$ continuous together with its second derivative, $\rho^{\prime}(x)<0$ for all $x \in \mathbb{R}, \rho( \pm \infty)=u_{ \pm}$, the following estimate holds:

$$
\inf _{x \in \mathbb{R}} F(\rho) \leq c \leq \sup _{x \in \mathbb{R}} F(\rho)
$$

where

$$
F(\rho)=\left(D \rho^{\prime \prime}+\int_{-\infty}^{\infty} \phi(x-y) S(\rho(y+a)) d y-k \rho\right) /\left(-\rho^{\prime}\right)
$$

Proof. Let us verify the right-hand side inequality in (2.4). Suppose that it does not hold. Then for some function $\rho(x)$, we have $c>\sup _{x \in \mathbb{R}} F(\rho)$. Then there exists such value $c_{0}$ that

$$
c>c_{0}>\sup _{x \in \mathbb{R}} F(\rho)
$$

and, consequently, the inequality

$$
D \rho^{\prime \prime}+c_{0} \rho^{\prime}+\int_{-\infty}^{\infty} \phi(x-y) S(\rho(y+a)) d y-k \rho<0
$$

holds for all $x \in \mathbb{R}$. Consider the Cauchy problem

$$
\frac{\partial v}{\partial t}=D \frac{\partial^{2} v}{\partial x^{2}}+c_{0} \frac{\partial v}{\partial x}+\int_{-\infty}^{\infty} \phi(x-y) S(v(y+a, t)) d y-k v
$$

with the initial condition $v(x, 0)=\rho(x)$. Then by virtue of the comparison theorems [7] and (2.5), the solution $v(x, t)$ decreases as a function of $t$ for every $x$. Hence, we get the estimate

$$
v(x, t) \leq \rho(x), \quad x \in \mathbb{R}, \quad t \geq 0 .
$$

Let $u(x, t)$ be solution of equation (1.1) with the same initial condition. Then $v(x, t)=$ $u\left(x+c_{0} t, t\right)$. Convergence (2.3) implies:

$$
\sup _{x \in \mathbb{R}}\left|v(x, t)-w\left(x-\left(c-c_{0}\right) t-h\right)\right|=\sup _{x \in \mathbb{R}}\left|u\left(x+c_{0} t, t\right)-w\left(x-\left(c-c_{0}\right) t-h\right)\right| \rightarrow 0, \quad t \rightarrow \infty .
$$


Since $c>c_{0}$ and $w(x)$ is a decreasing function, then $v(x, t) \rightarrow u_{-}$for each $x$ fixed, as $t \rightarrow \infty$. This convergence contradicts (2.7). This contradiction proves the right inequality in (2.4). The left inequality can be proved similarly.

Theorem 2.2. Suppose that $\tau=0, S(u)$ is increasing, and there exists a monotonically decreasing solution $w(x)$ of problem $(2.1),(2.2)$. Then

$$
c=\inf _{\rho} \sup _{x} F(\rho)=\sup _{\rho} \inf _{x} F(\rho),
$$

where infimum in the first equality and supremum in the second equality are taken with respect to all functions $\rho(x)$ continuous together with their second derivatives, and such that $\rho^{\prime}(x)<0$ for all $x \in \mathbb{R}, \rho( \pm \infty)=u_{ \pm}$.

Proof. The inequalities

$$
\sup _{\rho} \inf _{x} F(\rho) \leq c \leq \inf _{\rho} \sup _{x} F(\rho)
$$

follow from (2.4). It remains to note that if we take the wave solution as a test function, $\rho(x) \equiv w(x)$, then we obtain the equalities.

Theorem 2.3. Suppose that $\tau>0, a=0, S(u)$ is increasing, and there exists a monotonically decreasing solution $w(x)$ of problem $(2.1),(2.2)$. Then

$$
c=\inf _{\rho} \sup _{x} \Phi(\rho, c)=\sup _{\rho} \inf _{x} \Phi(\rho, c)
$$

where

$$
\Phi(\rho, c)=\left(D \rho^{\prime \prime}+\int_{-\infty}^{\infty} \phi(x-y) S(\rho(y+c \tau)) d y-k \rho\right) /\left(-\rho^{\prime}\right)
$$

This equation has a unique solution.

Proof. Set $a=\sigma \tau$ in (2.9), and denote by $c(\sigma)$ the left-hand side of this equality in order to show its dependence on $\sigma$. Then

$$
c(\sigma)=\inf _{\rho} \sup _{x} \Phi(\rho, \sigma)=\sup _{\rho} \inf _{x} \Phi(\rho, \sigma)
$$

since $\Phi(\rho, \sigma)=F(\rho)$ (for $a=\sigma \tau)$. Since $\rho(x)$ is a decreasing function, then for any $\rho$ fixed and for any $x \in \mathbb{R}, \Phi(\rho, \sigma)(x)$ is a non-increasing function of $\sigma$. Hence $c(\sigma)$ is a nonincreasing continuous function of $\sigma$. Therefore, equation $c(\sigma)=\sigma$ has a unique solution. It provides equality (2.10) with $c=\sigma$. 


\section{Non-monotone case}

The results presented above essentially use the wave monotonicity and global stability. They are obtained in the case where the function $S(u)$ is monotonically increasing and the function $\phi(x)$ is non-negative. These assumptions provide the applicability of the maximum principle and comparison theorems for equation (1.1). We will now obtain some estimates of the wave speed for a non-monotone function $S(u)$. The case of $\phi(x)$ with a variable sign can be studied similarly.

Lemma 3.1. Let $S(u) \leq S_{+}(u)$ for all $u \geq 0$ and some smooth monotonically increasing function $S_{+}(u)$. Assume, moreover, that $\phi(x) \geq 0$. Then the solution $v(x, t)$ of the equation

$$
\frac{\partial v}{\partial t}=D \frac{\partial^{2} v}{\partial x^{2}}+\int_{-\infty}^{\infty} \phi(x-y) S_{+}(v(y+a, t-\tau)) d y-k v
$$

with an initial condition $v(x, t)=v_{0}(x, t),-\tau \leq t \leq 0$ satisfies the inequality

$$
v(x, t) \geq u(x, t),
$$

where $u(x, t)$ is the solution of equation (1.1) with the same initial condition.

Proof. By virtue of the conditions on the functions $S_{+}$and $S$, we have

$$
S_{+}(v)-S(u)=\left(S_{+}(v)-S_{+}(u)\right)+\left(S_{+}(u)-S(u)\right)=b(x, t)(v-u)+g(x, t),
$$

where $b(x, t)=0$ if $v(x, t)=u(x, t)$ and

$$
b(x, t)=\frac{S_{+}(v)-S_{+}(u)}{v-u} \geq 0
$$

otherwise, $g(x, t)=S_{+}(u)-S(u) \geq 0$. Set $z=v-u$ and subtract equation (1.1) from equation (3.1). We get

$$
\frac{\partial z}{\partial t}=D \frac{\partial^{2} z}{\partial x^{2}}+\int_{-\infty}^{\infty} \phi(x-y)(b(y, t) z(y+a, t-\tau)+g(y, t)) d y-k z .
$$

Since $z(x, t)=0$ for $-\tau \leq t \leq 0$, then $z(x, t) \geq 0$ for all $t \geq 0$. The lemma is proved.

Suppose, next, that $S(u) \geq S_{-}(u)$ for $u \geq u$, where $S_{-}(u)$ is a monotonically increasing function. Then a similar assertion holds with inequality (3.2) replaced by an opposite inequality.

Theorem 3.2. Suppose that $S_{-}(u) \leq S(u) \leq S_{+}(u)$ for $u \geq 0$, where $S_{-}(u)$ and $S_{+}(u)$ are smooth monotonically increasing functions. Suppose, next, that each of the equations

$$
S(u)=k u, \quad S_{-}(0)=k u, \quad S_{+}(u)=k u
$$


has three solutions: $u_{ \pm}, u_{0} ; u_{ \pm}^{(+)}, u_{0}^{(+)} ; u_{ \pm}^{(-)}, u_{0}^{(-)}$, respectively, such that

$$
\begin{gathered}
u_{+}<u_{0}<u_{-}, \quad u_{+}^{(+)}<u_{0}^{(+)}<u_{-}^{(+)}, \quad u_{+}^{(-)}<u_{0}^{(-)}<u_{-}^{(-)}, \\
S^{\prime}\left(u_{ \pm}\right)<k, \quad S_{ \pm}^{\prime}\left(u_{ \pm}^{( \pm)}\right)<k
\end{gathered}
$$

and $u_{+}<u_{0}^{( \pm)}<u_{-}$.

Assume that problem (2.1), (2.2) has a travelling wave solution with a speed $c$. Then the following estimate holds:

$$
c_{-} \leq c \leq c_{+}
$$

where $c_{ \pm}$are the wave speed in the problems

$$
\begin{gathered}
D w^{\prime \prime}+c w^{\prime}+\int_{-\infty}^{\infty} \phi(x-y) S_{ \pm}(w(y+a+c \tau)) d y-k w=0, \\
w( \pm \infty)=u_{ \pm}^{( \pm)} .
\end{gathered}
$$

Proof. Consider equation (3.1) with the initial condition $v(x, t)=w(x),-\tau \leq t \leq 0$, where $w(x)$ is a solution of problem $(2.1),(2.2)$. The solution of this problem converges to the travelling wave solution, that is, solution of problem (3.5), (3.6) with $c=c_{+}$. From Lemma 3.1 it follows that $c \leq c_{+}$. The left-hand side inequality in (3.4) can be proved similarly.

\section{Estimates of the wave speed}

\subsection{Analytical solution}

We will now apply the minimax representation in order to obtain analytical estimates of the wave speed. Accuracy of the estimates depends on the choice of test functions $\rho(x)$. If the estimates from above and from below are close to each other, then they are also close to the exact value of the speed. We will construct test functions as exact solutions of some approximate models. Let

$$
S_{0}(u)= \begin{cases}0 & , \quad u<u_{0} \\ b & , \quad u \geq u_{0}\end{cases}
$$

and $\phi(x)=\phi_{0} \exp (-r|x|)$ for some positive constants $u_{0}, b, \phi_{0}$, and $r, k u_{0}<b$. In this case, equation (2.1) $(a=\tau=0)$ can be solved explicitly. In order to simplify the formulas below, we set $\phi_{0}=r$. If $w(x)$ is a decreasing function and $w(0)=u_{0}$, then

$$
J(w) \equiv \int_{-\infty}^{\infty} \phi(x-y) S_{0}(w(y)) d y=b\left(2-e^{r x}\right), x<0, \quad J(w)=b e^{-r x}, x \geq 0 .
$$


Equation (2.1) is reduced to the problem

$$
\begin{gathered}
D w^{\prime \prime}+c w^{\prime}-k w+b\left(2-e^{r x}\right)=0, x<0, \quad D w^{\prime \prime}+c w^{\prime}-k w+b e^{-r x}=0, x \geq 0, \\
w(-0)=w(+0), \quad w^{\prime}(-0)=w^{\prime}(+0) .
\end{gathered}
$$

If $D r^{2} \pm c r \neq k$, its solution writes:

$$
w(x)=c_{1} e^{\mu_{1} x}+c_{2} e^{r x}+\frac{2 b}{k}, x \leq 0, \quad w(x)=c_{3} e^{\mu_{2} x}+c_{4} e^{-r x}, x \geq 0,
$$

where

$$
\begin{gathered}
\mu_{1}=-\frac{c}{2 D}+\sqrt{\frac{c^{2}}{4 D^{2}}+\frac{k}{D}}, \quad \mu_{2}=-\frac{c}{2 D}-\sqrt{\frac{c^{2}}{4 D^{2}}+\frac{k}{D}}, \quad c_{1}=\frac{\alpha \mu_{2}-\beta}{\mu_{2}-\mu_{1}}, \quad c_{3}=\frac{\alpha \mu_{1}-\beta}{\mu_{2}-\mu_{1}}, \\
c_{2}=\frac{b}{\left(D r^{2}+c r-k\right)}, \quad c_{4}=-\frac{b}{\left(D r^{2}-c r-k\right)}, \quad \alpha=c_{4}-c_{2}-\frac{2 b}{k}, \quad \beta=-\left(c_{2}+c_{4}\right) r .
\end{gathered}
$$

Condition $w(0)=u_{0}$ provides the equation for the wave speed $c=c_{0}$ :

$$
\frac{\alpha \mu_{1}-\beta}{\mu_{2}-\mu_{1}}-\frac{b}{\left(D r^{2}-c r-k\right)}=u_{0}
$$

In the limiting case $D=0$, after solving (4.4), we obtain the expression for the wave speed:

$$
c_{0}=\frac{1}{r}\left(\frac{b \phi_{0}}{r u_{0}}-k\right) .
$$

In order to compare with numerical simulations, this formula is given for the case where $\phi_{0}$ can be different from $r$.

In the limiting case of large $r\left(\phi_{0}=r\right)$, which corresponds to the local equation, after solving (4.4), we obtain:

$$
c_{0}=\frac{2 \sigma \sqrt{k D}}{\sqrt{1-\sigma^{2}}}, \quad \sigma=1-\frac{k u_{0}}{b} .
$$

Numerical simulations of equation (1.1) with the step-wise constant function $S_{0}(u)$ and an exponential function $\phi(x)$ are shown in Figure 1. In the case of small diffusion coefficients (curves 1 and 2), the wave speed practically coincides with the analytical formula (4.5) obtained for $D=0$. The influence of the diffusion coefficient becomes more essential for $D=10^{-3}$ and $D=10^{-2}$. It is interesting to note that the dependence of the wave speed on diffusion coefficient for the integro-differential equation is quite different in comparison with 

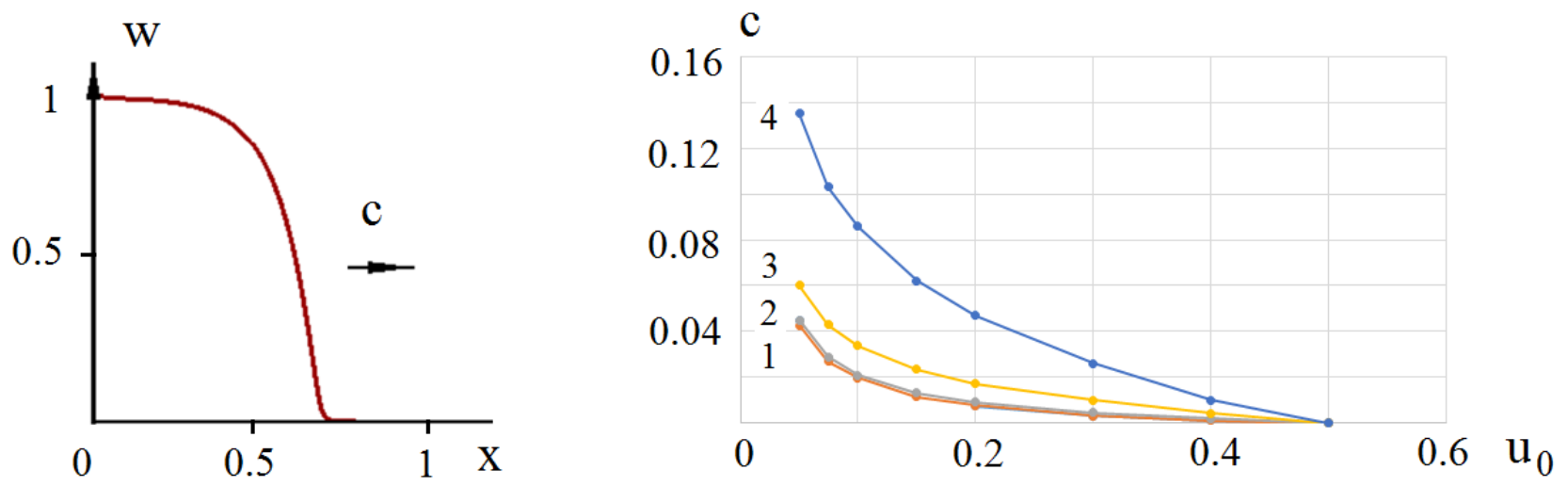

Figure 1: Numerical simulation of wave propagation. A snapshot of solution (left) and the wave speed dependence on the parameter $u_{0}$ (right) found numerically and by the analytical formula (4.5). The curves are obtained for different values of the diffusion coefficient: 1 . $D=10^{-6}, 2 . D=10^{-4}, 3 . D=10^{-3}, 4 . D=10^{-2}$. The values of other parameters are as follows: $k=0.1, b=0.1, r=20, \phi_{0}=10$. The analytical curve coincides with curve 1 .

the reaction-diffusion equation where the wave speed grows proportionally to $\sqrt{D}$ (see also Figure 2). Numerical simulations were carried out with an implicit finite difference method based on the Tomas algorithm for the inversion of the tridiagonal matrix. Numerical accuracy was verified by the convergence of the results under the decrease of time and space steps and by the comparison with the analytical results.

\subsection{Estimates and asymptotics}

Consider now a smooth increasing function $S(u)$ such that $S(u) \equiv S_{0}(u)$ for $\left|u-u_{0}\right| \geq \epsilon$. We will use the estimate of the wave speed (Proposition 2.2) with the test function $w(x)$ given by (4.3). We have

$$
F(w)=c_{0}+H(x)
$$

where

$$
H(x)=\frac{1}{w^{\prime}(x)} \int_{-\infty}^{\infty} \phi(x-y)\left(S_{0}(w(y))-S(w(y))\right) d y
$$

Hence

$$
c_{0}+\inf _{x} H(x) \leq c \leq c_{0}+\sup _{x} H(x) .
$$

Since $w(0)=u_{0}$ and $\left|w(y)-u_{0}\right| \geq \epsilon$ for $|y| \geq \epsilon / \beta$, where $\beta \sim\left|w^{\prime}(0)\right|$, then 


$$
|H(x)| \leq \frac{b}{\left|w^{\prime}(x)\right|} \int_{-\epsilon / \beta}^{\epsilon / \beta} \phi(x-y) d y .
$$

The right-hand side of the last inequality converges to 0 as $\epsilon \rightarrow 0$ if the function $\phi(x)$ has, for example, a bounded support or decays at infinity faster than $w^{\prime}(x)$. Thus, inequalities (4.7) provide not only the estimates of the wave speed but also its convergence to $c_{0}$ as $\epsilon \rightarrow 0$.

We finally consider one more numerical example with the function

$$
S(u)=\frac{2 b}{\pi} \arctan \left(\left(u-u_{0}\right) / \epsilon\right)+b
$$

in order to illustrate asymptotics of the wave speed. Figure 2 (left) shows the dependence of the wave speed on $\epsilon$. The limiting value for $\epsilon \rightarrow 0$ coincides with the value found for $S_{0}(u)$.
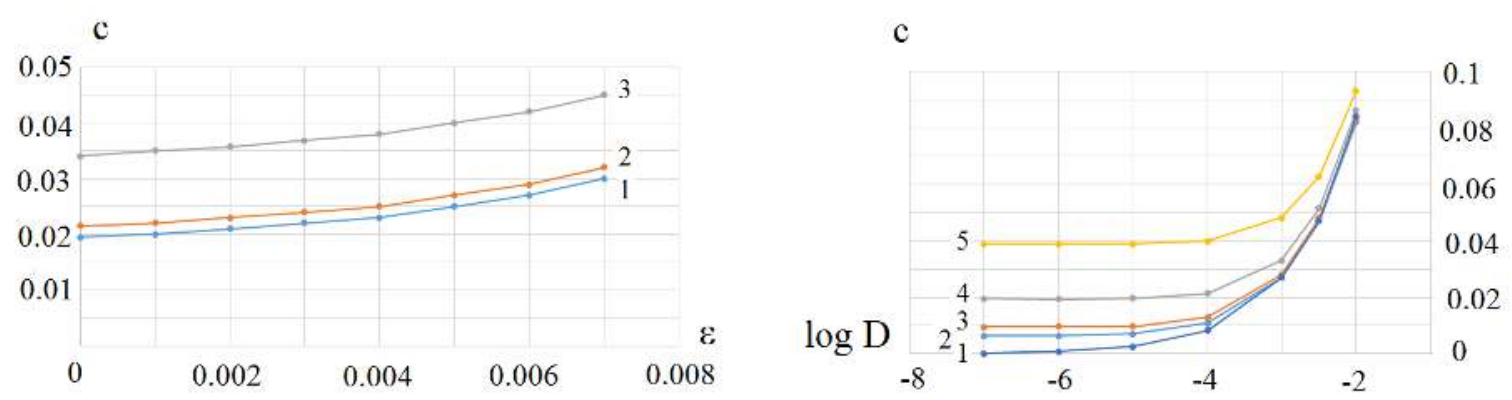

Figure 2: Dependence of the wave speed on $\epsilon$ (left) and on $D$ (right). The values of parameters are as follows: $k=0.1, r=20, \phi_{0}=10, b=0.05, u_{0}=0.1,1 . D=10^{-6}, 2 . D=10^{-4}, 3$. $D=10^{-3}$ (left); $k=0.1, b=0.05, u_{0}=0.1, \epsilon=10^{-5}, r=2 \phi_{0} ; 1$. analytical formula (4.6), 2 . $\phi_{0}=30,3 . \phi_{0}=20,4 . \phi_{0}=10,5 . \phi_{0}=5$ (right).

The dependence of the wave speed on the diffusion coefficient $D$ for different values of $r$ is presented in Figure 2 (right). Variation of $r$ (with the corresponding change of $\phi_{0}$ ) determines the value of nonlocal interaction. The limit of large $r$ corresponds to the local equation. This figure clearly shows the presence of two different mechanisms of wave propagation. The first one corresponds to sufficiently small values of the diffusion coefficient. The wave speed is practically independent of $D$ but it depends on the range of nonlocal interaction $r$. It can be called nonlocal regime of wave propagation. The second mechanism is determined by diffusion. It is observed for sufficiently large values of $D$. The wave speed depends on $D$ as a square root, and it weakly depends on $r$. This can be called diffusion regime of wave propagation. The wave speed in the nonlocal regime is well approximated by formula (4.5) and in the diffusion regime by formula (4.6). 


\section{Conclusions}

Integro-differential equations describe various processes in biological and biomedical applications. In many cases, these equations have travelling wave solutions. The speed of wave propagation characterizes these processes. In particular, the speed of cortex waves should belong to a certain range providing normal brain functioning. It depends on the response and connectivity functions of populations of neurons. In the damaged cortical tissue, these parameters can change following a stroke for example, resulting in a decrease of the cortical wave speed. External stimulation with the characteristics calculated from the properties of healthy and partially damaged cortical tissue can be introduced in order to restore the wave speed.

Though several methods are developed to determine the speed of wave propagation for reaction-diffusion equations, we are not aware whether other representations are applicable for the problem under consideration. The estimates obtained in this work are applicable under some restrictive conditions on the function $S(u)$. Further studies are necessary to generalize them for a broader class of functions.

\section{Acknowledgments}

N. Bessonov was supported by Russian Foundation of Basic Research grant 16-01-00068, 2016-2018. S. Trofimchuk was supported by FONDECYT (Chile) project 1150480, and V. Volpert by the "RUDN University Program 5-100".

\section{References}

[1] B. Ermentrout, J.B. McLeod. Existence and uniqueness of traveling waves for a neural network. Proc. Roy. Soc. Edinburgh, 123A (1993), 461-478.

[2] J. Modolo, B. Bhattacharya, R. Edwards, J. Campagnaud, A. Legros, A. Beuter. Using a virtual cortical module implementing a neural field model to modulate brain rhythms in Parkinsons disease Frontiers in Neuroscience, 4 (2010), article 45.

[3] K. P. Hadeler, F. Rothe, Travelling fronts in nonlinear diffusion equations, J. Math. Biol. 2 (1975), 251263.

[4] Vit. A. Volpert, Vl. A. Volpert. Propagation velocity estimation for condensed phase combustion. SIAM J. Appl. Math., 51 (1991), no. 4, 1074-1089.

[5] V. Volpert. Elliptic partial differential equations. Volume 2. Reaction-diffusion equations. Birkhauser, Basel, 2014.

[6] T. Galochkina, A. Bouchnita, P. Kurbatova, V. Volpert. Reaction-diffusion waves of blood coagulation. Mathematical Biosciences, 2017, 288 (2017), 130-139. 
[7] A. Ducrot, M. Marion, V. Volpert. Spectrum of some integro-differential operators and stability of travelling waves. Nonlinear Analysis Series A: Theory, Methods and Applications, 74 (2011), no. 13, 4455-4473.

[8] J. Coville, L. Dupaigne. Propagation speed of travelling fronts in non local reactiondiffusion equations. Nonlinear Anal. 60 (2005), no. 5, 797-819.

[9] R.D. Benguria, M.C. Depassier. Variational characterization of the speed of propagation of fronts for the nonlinear diffusion equation. Comm. Math. Phys. 175 (1996) 221-227.

[10] S. Heinze, G. Papanicolaou, A. Stevens. Variational principles for propagation speeds in inhomogeneous media. SIAM J. Appl. Math. 62 (2001), no. 1, 129-148.

[11] X. Chen. Existence, uniqueness, and asymptotic stability of travelling waves in nonlocal evolution equations. Adv. Differential Equations, 1997, 2, 125-160.

[12] S. Ma, J. Wu. Existence, uniqueness and asymptotic stability of traveling wavefronts in a non-local delayed diffusion equation. Journal of Dynamics and Differential Equations, 19 (2007), No. 2, 391-436. 